\title{
ANALYSIS OF THE DISCRETE ORNSTEIN-UHLENBECK PROCESS CAUSED BY THE TICK SIZE EFFECT
}

DANIEL WEI-CHUNG MIAO, ${ }^{*}$ National Taiwan University of Science and Technology

\begin{abstract}
This paper provides an analysis on a discrete version of the Ornstein-Uhlenbeck (OU) process which reflects the small discrete movements caused by the tick size effect. This discrete OU process is derived from matching the first two moments to those of the standard OU process in an infinitesimal sense. We discuss the distributional convergence from the discrete to the continuous processes, and show that the convergence speed is in the second order of the step (tick) size. We also provide some analytical results for the proposed discrete OU process itself, including the closed-form formula of the moment generating function and a full characterisation of the steady state distribution. These results enable us to examine the convergence order explicitly.
\end{abstract}

Keywords: Discrete Ornstein-Uhlenbeck process; moment matching; tick size; convergence order; moment generating function; steady state

2010 Mathematics Subject Classification: Primary 60G20; 60J27

Secondary 60G15; 60J75

\section{Introduction}

Many standard financial models are stochastic processes where time and state space are both continuous. Such processes use Brownian motions to drive the random movements of the concerned financial quantities. For example, the geometric Brownian motions are used to model stock prices in the celebrated Black-Scholes model [1], while the Ornstein-Uhlenbeck processes [11] are used to describe those quantities with a mean-reverting nature such as interest rates or foreign exchange rates. Well known examples for the latter are the Vasiček model [12] and its further extension by Hull and White [6]. The continuous time assumption can be justified by the fact that trading takes place at any real time during an ordinary market open day. However, the continuous state assumption is somewhat restrictive in that the changes in these quantities (stock prices, interest rates, etc.) are limited by the tick size, the minimal unit by which they may change. In other words, for these quantities the states actually change in a discrete manner and the continuous state models are only an approximation.

If the tick size becomes greater, the error between discrete and continuous state models will be more significant. In addition to the error caused, there is a notable difference between the paths of both processes. Consider an inactive market where little trading takes place; the asset price may stay at a constant level for a certain while until a new trade comes in and causes a price change. This is not normally seen in the continuous state models driven by Brownian motions since their state variables change continuously over time. For this kind of random path, more appropriate models should have their states changing discretely within a countable

Received 1 December 2011; revision received 1 October 2012.

* Current address: Imperial College Business School, Tanaka Building, South Kensington Campus, London SW7 2AZ, UK. Email address: miao@mail.ntust.edu.tw 
set of state values and the changes should happen in continuous time. The state should move up or down to the neighbouring states such that it changes sequentially within these discrete values.

This paper intends to discuss the effect of the discrete nature caused by the tick size. The continuous state (and time) model is seen as a limiting case, of which its discrete state counterpart (time is still continuous) is developed to capture the tick size effect. The research target chosen in this study is the OU process which is suitable for the modelling of mean-reverting financial processes. Its discrete counterpart is constructed by matching the first two moments across an infinitesimal interval to those of the original OU process. The resultant process is therefore a continuous time Markov chain that moves within a discrete set and exhibits the mean-reverting nature.

Existing studies on the discrete OU processes are limited, and most of them are concerned with processes which have discrete time and state. A discretisation in this sense leads to a discrete time Markov chain, for which some results can be found in the literature. For example, Renshaw [10] considered the correlated random walk with friction as a discrete OU process and derived the expressions for its moment generating function and some statistics including variance and kurtosis. Larralde [7] studied the first passage time distribution while Lefebvre and Guilbault [9] studied the first hitting place probabilities. Larralde [8] discussed statistical properties and characteristic functions of time and state discrete OU processes.

The purpose of our study is two-fold. On the one hand, we intend to examine the order of convergence from the discrete to the continuous processes. It is shown that the convergence is in the second order of step size as it tends to zero. In this regard, our study is similar to other studies on the convergence order of different models, e.g. [2] and [13], where convergence from various tree models to the Black-Scholes model is discussed. On the other hand, we intend to derive two analytical results for the discrete OU process itself. The first result is the closed-form formula of the moment generating function (MGF) at an arbitrary time horizon including steady state. This is derived by solving the first order PDE for the MGF. Secondly, we give a full characterisation of the steady state probability distribution. This is derived from equilibrium, a steady state property that makes the Markov chain particularly tractable. The convergence order can also be checked from these analytical results.

The rest of this paper is organised as follows. Section 2 introduces our version of the discrete OU process and discusses its fundamental properties. Section 3 conducts the convergence analysis which leads to second order convergence in distribution. Section 4 derives the closedform formula of the MGF and examines its convergence order. Section 5 gives a full characterisation of the probability distribution at steady state. Finally, Section 6 gives conclusions.

\section{Construction of the discrete OU process}

Consider the continuous OU process which solves the following stochastic differential equation

$$
\mathrm{d} X_{t}=k\left(\theta-X_{t}\right) \mathrm{d} t+\sigma \mathrm{d} W_{t}, \quad X_{0}=x_{0},
$$

with $k, \theta$, and $\sigma$ being the speed of mean reversion, long run mean, and volatility, respectively. Suppose that $X_{t}=x$ and let $\Delta t$ be an infinitesimal time interval; the first two moments of $X_{t+\Delta t}$ are given by

$$
\begin{gathered}
\mathbb{E}\left[X_{t+\Delta t} \mid X_{t}=x\right]=x+k(\theta-x) \Delta t+o(\Delta t), \\
\mathbb{E}\left[X_{t+\Delta t}^{2} \mid X_{t}=x\right]=\sigma^{2} \Delta t+\left[x^{2}+2 x k(\theta-x) \Delta t\right]+o(\Delta t) .
\end{gathered}
$$


Our purpose here is to define a discrete OU process, denoted as $X_{t}^{h}$ where $h$ is the step size in space (tick size), that carries the main properties of $X_{t}$. Let $X$ be the discrete set of the state values of $X_{t}^{h}$. Since the OU process is symmetric about the long run mean $\theta$, it is natural to pick $2 N+1$ states, i.e. $\mathcal{X}=\left\{x_{i}, i=1, \ldots, 2 N+1\right\}$, where $x_{i}<x_{i+1}, h=x_{i+1}-x_{i}$, and the center state is $x_{N+1}=\theta$. The boundary states are $x_{1}=\theta-N h$ and $x_{2 N+1}=\theta+N h$, and

$$
x_{i}=x_{1}+(i-1) h=\theta-(N+1-i) h, \quad i=1, \ldots, 2 N+1 .
$$

For convenience, we assume that both processes start from the same initial value, i.e. $X_{0}^{h}=$ $X_{0}=x_{0} \in \mathcal{X}$.

To capture the continuity in the path of $X_{t}$, the discrete OU process $X_{t}^{h}$ is only allowed to jump to adjacent states, i.e. one step up or down. For each state $x_{i}$, to determine the up and down jump rates $u_{i}$ and $d_{i}$ for which $X_{t}^{h}$ behaves in a way close to $X_{t}$, we match their first two moments across the time interval $[t, t+\Delta t]$. Namely, for all $t>0$ and $x_{i} \in \mathcal{X}$, we demand

$$
\mathbb{E}\left[\left(X_{t+\Delta t}^{h}\right)^{n} \mid X_{t}^{h}=x_{i}\right]=\mathbb{E}\left[X_{t+\Delta t}^{n} \mid X_{t}=x_{i}\right], \quad n=1,2 .
$$

Letting $\Delta t \rightarrow 0$, this moment matching is actually done in an infinitesimal sense.

We now derive the formulas for the up and down jump rates $u_{i}$ and $d_{i}$. Conditional on $X_{t}^{h}=x_{i}$, because $\mathbb{P}\left(X_{t+\Delta t}^{h}=x_{i}+h \mid X_{t}^{h}=x_{i}\right)=u_{i} \Delta t+o(\Delta t)$ and $\mathbb{P}\left(X_{t+\Delta t}^{h}=x_{i}-\right.$ $\left.h \mid X_{t}^{h}=x_{i}\right)=d_{i} \Delta t+o(\Delta t)$, the first two moments of $X_{t+\Delta t}^{h}$ are

$$
\begin{aligned}
& \mathbb{E}\left[X_{t+\Delta t}^{h} \mid X_{t}^{h}=x_{i}\right] \\
& \quad=u_{i} \Delta t\left(x_{i}+h\right)+\left(1-\left(u_{i}+d_{i}\right) \Delta t\right) x_{i}+d_{i} \Delta t\left(x_{i}-h\right)+o(\Delta t), \\
& \mathbb{E}\left[\left(X_{t+\Delta t}^{h}\right)^{2} \mid X_{t}^{h}=x_{i}\right] \\
& \quad=u_{i} \Delta t\left(x_{i}+h\right)^{2}+\left(1-\left(u_{i}+d_{i}\right) \Delta t\right) x_{i}^{2}+d_{i} \Delta t\left(x_{i}-h\right)^{2}+o(\Delta t) .
\end{aligned}
$$

Matching (3) to (1) and ignoring the $o(\Delta t)$ terms, we obtain

$$
u_{i}=\frac{\sigma^{2}+h k\left(\theta-x_{i}\right)}{2 h^{2}}, \quad d_{i}=\frac{\sigma^{2}-h k\left(\theta-x_{i}\right)}{2 h^{2}} .
$$

It is seen from (4) that $u_{i}<d_{i}$ when $x_{i}>\theta$ and vice versa, simply reflecting the mean-reverting property. Moreover, due to the uniform step size $h$ in $\left\{x_{i}\right\},\left\{u_{i}\right\}$ is a decreasing arithmetic sequence, $\left\{d_{i}\right\}$ is an increasing arithmetic sequence, and we have $\Delta u\left(=u_{i}-u_{i+1}\right)=\Delta d(=$ $\left.d_{i+1}-d_{i}\right)=k / 2$.

Although $X_{t}$ can take any real value, its variance will not go to infinity as time advances since the process is mean-reverting. This ensures the existence of a steady state when the probability distribution does not change over time. Therefore, using a finite set $X$ for $X_{t}^{h}$ should be sufficient to approximate $X_{t}$ well, as long as the probability of $X_{t}$ going beyond the range $\left[x_{\min }, x_{\max }\right]$ (where $x_{\min }=x_{1}$ and $x_{\max }=x_{2 N+1}$ are the minimal and maximal values of $\mathcal{X}$ ) is negligible. Since $X_{t}^{h}$ cannot jump over these boundaries, we shall also demand that $d_{1}=0$ and $u_{2 N+1}=0$. This condition gives the relationship between $N$ and $h$ as

$$
N=\frac{\sigma^{2}}{h^{2} k} \quad \text { or } \quad h=\frac{\sigma}{\sqrt{N k}} .
$$

Since $N$ is an integer, only particular choices of $h$ will make this equality hold. For convenience we only consider such values of $h$. For a given pair of $N$ and $h$, the boundaries of $\mathcal{X}$ are

$$
\left[x_{\min }, x_{\max }\right]=[\theta-N h, \theta+N h]=\left[\theta-\frac{\sigma^{2}}{h k}, \theta+\frac{\sigma^{2}}{h k}\right] \text {. }
$$


As $N \rightarrow \infty$, the step size $h \rightarrow 0$ and $\left[x_{\min }, x_{\max }\right] \rightarrow(-\infty,+\infty)$, meaning that $X_{t}^{h}$ tends to cover the whole real line just like $X_{t}$.

Remark 1. Note that for a given $h$, the state space $\mathcal{X}$ is a finite set instead of a countable one. This is because one cannot find sensible $u_{i} \geq 0$ and $d_{i} \geq 0$ from (4) if the state value $x_{i} \notin\left[x_{\min }, x_{\max }\right]$. Hence, $\mathcal{X}$ must be upper and lower bounded for a fixed $h$, and will only become unbounded in the limiting case when $h \rightarrow 0$ and $\left[x_{\min }, x_{\max }\right] \rightarrow(-\infty,+\infty)$.

Remark 2. In the above construction we choose $\theta \in \mathcal{X}$ for convenience, but this is not strictly necessary. If $\theta \notin X$ and $\theta$ is still the centre of $\mathcal{X}$ (symmetric case, $\theta=\left(x_{j}+x_{j+1}\right) / 2$ for some $j$ where $x_{j}, x_{j+1} \in \mathcal{X}$ ), one may still follow the above procedure to find $u_{i}$ and $d_{i}$ for each state $x_{i}$, and $X_{t}^{h}$ is well defined. However, if $\theta \notin \mathcal{X}$ but $\theta$ is not at the centre (asymmetric case, $\left.\theta \neq\left(x_{j}+x_{j+1}\right) / 2\right)$, one may not find $u_{i}$ and $d_{i}$ for which (4) is satisfied for each state and $d_{\min }=u_{\max }=0$. In this case, $X_{t}^{h}$ is not well defined.

Since $X_{t}^{h}$ is upper and lower bounded for a fixed $h$, it will serve as a good approximation for $X_{t}$ only if the probability of $X_{t}$ moving out of this range is sufficiently small. The following proposition shows that this is the case.

Proposition 1. Consider an OU process $X_{t}$ starting from $x_{0} \in \mathcal{X}$. As $h \rightarrow 0$ the probability of $X_{t}$ moving out of $\left[x_{\min }, x_{\max }\right]$ converges to 0 at a speed quicker than $f(h)=\mathrm{e}^{-2 / h^{2}}$, i.e.

$$
\lim _{h \rightarrow 0} \frac{\mathbb{P}\left(X_{t} \notin\left[x_{\min }, x_{\max }\right]\right)}{f(h)}=0 .
$$

Proof. When the process is at steady state $(t \rightarrow \infty), X_{t}$ is normally distributed with mean $\theta$ and variance $\sigma^{2} / 2 k$. Therefore, $\mathbb{P}\left(X_{t} \notin\left[x_{\min }, x_{\max }\right]\right)=2 \mathbb{P}\left(X_{t}<\theta-\sigma^{2} / h k\right)=2 \Phi(-2 / h)$, where $\Phi(x)=\int_{-\infty}^{x} \phi(y) \mathrm{d} y$ and $\phi(x)=\mathrm{e}^{-x^{2} / 2} / \sqrt{2 \pi}$. Using l'Hopital's rule, we have

$$
\lim _{h \rightarrow 0} \frac{\mathbb{P}\left(X_{t} \notin\left[x_{\min }, x_{\max }\right]\right)}{f(h)}=\lim _{h \rightarrow 0} \frac{2 \Phi(-2 / h)}{\mathrm{e}^{-2 / h^{2}}}=\lim _{h \rightarrow 0} \frac{4 h^{-2} \phi(-2 / h)}{4 h^{-3} \mathrm{e}^{-2 / h^{2}}}=\lim _{h \rightarrow 0} \frac{h}{\sqrt{2 \pi}}=0 .
$$

Next, when the process is not at steady state $(t<\infty), X_{t}$ will follow a normal distribution with slightly different mean $\theta+\left(x_{0}-\theta\right) \mathrm{e}^{-k t}$ and variance $\sigma^{2}\left(1-\mathrm{e}^{-2 k t}\right) / 2 k$ (see [12]). The tail property of its distribution will not be affected by the slight changes in parameters. Hence, as $h \rightarrow 0, \mathbb{P}\left(\left.X_{t}\right|_{t<\infty} \notin\left[x_{\min }, x_{\max }\right]\right)$ should converge to 0 at the same speed as $\mathbb{P}\left(X_{t} \mid{ }_{t \rightarrow \infty} \notin\left[x_{\min }, x_{\max }\right]\right)$, i.e. the claimed property remains true.

Since the convergence speed of $\mathbb{P}\left(X_{t} \notin\left[x_{\min }, x_{\max }\right]\right)$ is quicker than that of an exponential function, it should be much quicker than that of any polynomial function with order $O\left(h^{n}\right)$, $n \in \mathbb{N}$. In contrast with $x_{\max }=O(1 / h)$ and $\left|x_{\min }\right|=O(1 / h)$ which grow to infinity in the first order of $1 / h, \mathbb{P}\left(X_{t} \notin\left[x_{\min }, x_{\max }\right]\right)$ goes to zero much more quickly than the growth of the range covered by $\left[x_{\min }, x_{\max }\right]$. This indicates that for small $h$ (large $\left.N\right), X_{t}^{h}$ should provide a good approximation of $X_{t}$.

Since the discrete OU process $X_{t}^{h}$ constructed above is a continuous time Markov chain, it is of interest to discuss some properties regarding its state holding times and state transition rates. For each state $x_{i}$, define

$$
\tau_{i}=\text { the state holding time at state } x_{i},
$$

$$
J_{i}(t)=\text { the number of (up and down) jumps during }[s, s+t] \text { if } X_{s}=x_{i} .
$$


Note that the random variables $\tau_{i}$ and $J_{i}(t)$ have no dependence on $i$ (state $x_{i}$ ), and therefore they can be written as $\tau$ and $J(t)$ (subscripts dropped). This is because $u_{i}+d_{i}=\sigma^{2} / h^{2}$ (see (4)) which indicates that the total rate for jumping out of any state $x_{i}$ does not depend on $x_{i}$. The following proposition gives more specific statements about their distributions.

Proposition 2. For the discrete OU process $X_{t}^{h}$ constructed above, the following statements hold.

1. For any $i$, the state holding time $\tau_{i}$ is exponentially distributed with parameter $\lambda=\sigma^{2} / h^{2}$. Namely, $\tau \sim \operatorname{Exp}\left(\sigma^{2} / h^{2}\right)$.

2. For any $i$, the number of jumps $J_{i}(t)$ is Poisson distributed with parameter $\lambda t=\sigma^{2} t / h^{2}$. Namely, $J(t) \sim \operatorname{Poi}\left(\sigma^{2} t / h^{2}\right)$.

The above proposition indicates that $J(t)$ is actually a Poisson process. The parameter $\lambda$ is interpreted as the jump arrival rate, and the holding time $\tau$ represents the interarrival time between successive jumps. Higher $\sigma$ and smaller $h$ will lead to larger $\lambda$ and make the resulting discrete process $X_{t}^{h}$ jump more frequently and have a shorter state holding time.

With the Markov chain $X_{t}^{h}$ well defined, the probability distribution at any time $t$ can be calculated. Let $\mathbf{p}_{h}(t)=\left[p_{i}^{h}(t), i=1, \ldots, 2 N+1\right]$ denote the probability vector of $X_{t}^{h}$ where $p_{i}^{h}(t)=\mathbb{P}\left(X_{t}^{h}=x_{i}\right)$. The Kolmogorov forward equations for the probabilities $p_{i}^{h}(t)$ are given as follows:

$$
\begin{aligned}
\frac{\mathrm{d}}{\mathrm{d} t} p_{1}^{h}(t) & =-p_{1}^{h}(t) u_{1}+p_{2}^{h}(t) d_{2}, & & i=1, \\
\frac{\mathrm{d}}{\mathrm{d} t} p_{i}^{h}(t) & =p_{i-1}^{h}(t) u_{i-1}-p_{i}^{h}(t)\left(u_{i}+d_{i}\right)+p_{i+1}^{h}(t) d_{i+1},, & & i=2, \ldots, 2 N, \\
\frac{\mathrm{d}}{\mathrm{d} t} p_{2 N+1}^{h}(t) & =p_{2 N}^{h}(t) u_{2 N}-p_{2 N+1}^{h}(t) d_{2 N+1}, & i & =2 N+1,
\end{aligned}
$$

or in matrix form as $\mathbf{p}_{h}^{\prime}(t)=\mathbf{p}_{h}(t) \mathbf{A}_{h}$, where the $(2 N+1) \times(2 N+1)$ matrix $\mathbf{A}_{h}$ is the generator matrix. The probability distribution at time $t$ can be obtained from $\mathbf{p}_{h}(t)=\mathbf{p}_{h}(0) \mathrm{e}^{\mathbf{A}_{h} t}$.

\section{Order of convergence}

As the discrete OU process $X_{t}^{h}$ is derived from the continuous OU process $X_{t}$, it is expected that, as the step size $h \rightarrow 0$, both of the processes will become identical. In this section we show that $X_{t}^{h}$ will converge to $X_{t}$ in distribution and derive the order of convergence.

Let $g(t, x)$ denote the probability density function (PDF) of $X_{t}$, i.e. $\mathbb{P}\left(X_{t} \in \mathrm{d} x \mid X_{0}=x_{0}\right)$ $=g(t, x) \mathrm{d} x$. Then $g(t, x)$ must satisfy the Kolmogorov forward equation or Fokker-Plank equation (see [3]) as below

$$
\frac{\partial g(t, x)}{\partial t}=-\frac{\partial}{\partial x}[k(\theta-x) g(t, x)]+\frac{1}{2} \frac{\partial^{2}}{\partial x^{2}}\left[\sigma^{2} g(t, x)\right],
$$

with an appropriate initial condition $g(0, x), x \in \mathbb{R}$. The probability distribution of the discrete process $X_{t}^{h}$ is described by its probability mass function as given in the probability vector $\mathbf{p}_{h}(t)$. In order to make the equations in (6) comparable with (7), we define $g_{h}\left(t, x_{i}\right)=p_{i}^{h}(t) / h$, 
$i=1, \ldots, 2 N+1$, and thus

$$
\begin{aligned}
\frac{\mathrm{d} g_{h}\left(t, x_{i}\right)}{\mathrm{d} t}= & g_{h}\left(t, x_{i-1}\right) u_{i-1}-g_{h}\left(t, x_{i}\right)\left(u_{i}+d_{i}\right)+g_{h}\left(t, x_{i+1}\right) d_{i+1} \\
= & g_{h}\left(t, x_{i-1}\right)\left[\frac{\sigma^{2}+h k\left(\theta-x_{i-1}\right)}{2 h^{2}}\right]-g_{h}\left(t, x_{i}\right)\left[\frac{\sigma^{2}}{h^{2}}\right] \\
& +g_{h}\left(t, x_{i+1}\right)\left[\frac{\sigma^{2}-h k\left(\theta-x_{i+1}\right)}{2 h^{2}}\right],
\end{aligned}
$$

where we let $u_{0}=d_{2 N+2}=0$. To relate (8) with (7), we apply the central differencing schemes to the first and second partial derivatives on the right-hand side of (7). This gives

$$
\begin{aligned}
\frac{\partial g\left(t, x_{i}\right)}{\partial t}= & -\left.\frac{\partial}{\partial x}[k(\theta-x) g(t, x)]\right|_{x=x_{i}}+\left.\frac{1}{2} \frac{\partial^{2}}{\partial x^{2}}\left[\sigma^{2} g(t, x)\right]\right|_{x=x_{i}} \\
= & -\left[\frac{k\left(\theta-x_{i+1}\right) g\left(t, x_{i+1}\right)-k\left(\theta-x_{i-1}\right) g\left(t, x_{i-1}\right)}{2 h}-T E_{1}\right] \\
& +\frac{1}{2}\left[\frac{\sigma^{2} g\left(t, x_{i+1}\right)-2 \sigma^{2} g\left(t, x_{i}\right)+\sigma^{2} g\left(t, x_{i-1}\right)}{h^{2}}-T E_{2}\right] \\
= & g\left(t, x_{i-1}\right)\left[\frac{\sigma^{2}+h k\left(\theta-x_{i-1}\right)}{2 h^{2}}\right]-g\left(t, x_{i}\right)\left[\frac{\sigma^{2}}{h^{2}}\right] \\
& +g\left(t, x_{i+1}\right)\left[\frac{\sigma^{2}-h k\left(\theta-x_{i+1}\right)}{2 h^{2}}\right]-T E,
\end{aligned}
$$

where $T E_{1}=\frac{1}{6}\left(\partial^{3} / \partial x^{3}\right)[\mu(x) g(t, x)] h^{2}+\cdots, T E_{2}=\frac{1}{12}\left(\partial^{4} / \partial x^{4}\right)\left[\sigma^{2}(x) g(t, x)\right] h^{2}+\cdots$, and $T E=-T E_{1}+\frac{1}{2} T E_{2}$ are truncation errors. Comparing (9) to (8), we see that these two equations differ only in the truncation error term $T E=O\left(h^{2}\right)$. This indicates that the effect of using moment matching to carry out the discretisation is essentially equivalent to applying central differencing schemes to the Kolmogorov forward equations.

As $h \rightarrow 0, T E \rightarrow 0$, i.e. the right-hand side of the ODE (8) converges to the right-hand side of the PDE (7) in second order. What we are concerned with is the convergence of the probability distribution, i.e. the convergence of the solution of (8) to that of (7). We will show that the convergence order seen in the differential equations (order of truncation errors) will carry over to their solutions.

Proposition 3. Suppose that the initial probability distributions of $X_{t}$ and $X_{t}^{h}$ satisfy $g\left(0, x_{i}\right)=$ $g_{h}\left(0, x_{i}\right)$ for all $x_{i} \in \mathcal{X}$. For any $t>0$ and $x_{i} \in \mathcal{X}$, as $h \rightarrow 0, g_{h}\left(t, x_{i}\right)$ converges to $g\left(t, x_{i}\right)$ in second order, i.e.

$$
g_{h}\left(t, x_{i}\right)-g\left(t, x_{i}\right)=O\left(h^{2}\right) .
$$

Proof. First we write (8) and (9) in matrix form as

$$
\begin{gathered}
\mathbf{g}_{h}^{\prime}(t)=\mathbf{g}_{h}(t) \mathbf{A}_{h}, \\
\mathbf{g}^{\prime}(t)=\mathbf{g}(t) \mathbf{A}_{h}-\mathbf{T E}_{h}(t),
\end{gathered}
$$

respectively, where we define the following $1 \times(2 N+1)$ row vectors $\mathbf{g}_{h}(t)=\left[g_{h}\left(t, x_{i}\right)\right]$, $\mathbf{g}(t)=\left[g\left(t, x_{i}\right)\right]$, and $\mathbf{T E}_{h}(t)=\left[T E_{h}^{i}(t)\right]$ for $i=1, \ldots, 2 N+1$. Subtracting the second equation from the first equation yields

$$
\mathbf{e}_{h}^{\prime}(t)=\mathbf{e}_{h}(t) \mathbf{A}_{h}+\mathbf{T E}_{h}(t),
$$


where $\mathbf{e}_{h}(t)=\mathbf{g}_{h}(t)-\mathbf{g}(t)$ containing elements $e_{h}\left(t, x_{i}\right)=g_{h}\left(t, x_{i}\right)-g\left(t, x_{i}\right)$. The solution to the above first order ODE system is given by

$$
\mathbf{e}_{h}(t)=\mathbf{e}_{h}(0) \mathrm{e}^{\mathbf{A}_{h} t}+\left[\int_{0}^{t} \mathbf{T E}_{h}(s) \mathrm{e}^{-\mathbf{A}_{h} s} \mathrm{~d} s\right] \mathrm{e}^{\mathbf{A}_{h} t} .
$$

For a row vector $\mathbf{x}=\left[x_{1}, \ldots, x_{n}\right]$, define the norm as $\|\mathbf{x}\|=\sum_{i=1}^{n}\left|x_{i}\right| h$. The corresponding norm for a matrix $\mathbf{B}$ is then defined by $\|\mathbf{B}\|=\max _{\|\mathbf{x}\|=1}\|\mathbf{x B}\|$. In our case we have $\left\|\mathbf{g}_{h}(t)\right\|=1$ since the elements of $\mathbf{g}_{h}(t)$ form a probability 'density' function (prob/h at each $x_{i}$ ). The matrices concerned are $\mathrm{e}^{\mathbf{A}_{h} t}$ or $\mathrm{e}^{-\mathbf{A}_{h} t}$. Note that $\mathrm{e}^{\mathbf{A}_{h} t}$ is the probability transition matrix across the interval $[0, t]$, i.e. $\mathbf{p}_{h}(t)=\mathbf{p}_{h}(0) \mathrm{e}^{\mathbf{A}_{h} t}$ or $\mathbf{p}_{h}(0)=\mathbf{p}_{h}(t) \mathrm{e}^{-\mathbf{A}_{h} t}$. If $\mathbf{x}$ is a vector of a probability density $(\|\mathbf{x}\|=1)$ like $\mathbf{g}_{h}(t)$, then $\mathbf{x e}^{\mathbf{A}_{h} t}$ and $\mathbf{x} \mathrm{e}^{-\mathbf{A}_{h} t}$ must also be vectors of probability densities. Therefore, we have

$$
\left\|\mathrm{e}^{\mathbf{A}_{h} t}\right\|=\max _{\|\mathbf{x}\|=1}\left\|\mathbf{x} \mathrm{e}^{\mathbf{A}_{h} t}\right\|=1 \quad \text { and } \quad\left\|\mathrm{e}^{-\mathbf{A}_{h} t}\right\|=\max _{\|\mathbf{x}\|=1}\left\|\mathbf{x} \mathrm{e}^{-\mathbf{A}_{h} t}\right\|=1 .
$$

Because $g_{h}\left(0, x_{i}\right)=g\left(0, x_{i}\right)$ for all $i$, i.e. $\mathbf{g}_{h}(0)=\mathbf{g}(0)$, the initial error $\mathbf{e}_{h}(0)=0$. Moreover, because the truncation error for each time $t>0$ and $x_{i} \in \mathcal{X}$ converges to 0 in second order, there exists a constant $c_{i}(t)$ such that $T E_{h}^{i}(t) \leq c_{i}(t) h^{2}$. Since $\left\|\mathbf{T E}_{h}(t)\right\|=$ $\sum_{i=1}^{2 N+1}\left|T E_{h}^{i}(t)\right| h$ with $N=\sigma^{2} / h^{2} k \propto 1 / h^{2},\left\|\mathbf{T E}_{h}(t)\right\|$ is first order in $h$, i.e. there exists a constant $c(t)$ such that $\left\|\mathbf{T E}_{h}(t)\right\| \leq c(t) h$. Taking the norm in (10), we obtain

$$
\begin{aligned}
\left\|\mathbf{e}_{h}(t)\right\| & =\left\|\mathbf{e}_{h}(0) \mathrm{e}^{\mathbf{A}_{h} t}\right\|+\left\|\left[\int_{0}^{t} \mathbf{T E}_{h}(s) \mathrm{e}^{-\mathbf{A}_{h} s} \mathrm{~d} s\right] \mathrm{e}^{\mathbf{A}_{h} t}\right\| \\
& \leq\left\|\mathbf{e}_{h}(0)\right\|\left\|\mathrm{e}^{\mathbf{A}_{h} t}\right\|+\left[\int_{0}^{t}\left\|\mathbf{T} \mathbf{E}_{h}(s)\right\|\left\|\mathrm{e}^{-\mathbf{A}_{h} s}\right\| \mathrm{d} s\right]\left\|\mathrm{e}^{\mathbf{A}_{h} t}\right\| \\
& \leq 0+\int_{0}^{t} c(s) h \mathrm{~d} s \\
& =C_{t} h \\
& =O(h)
\end{aligned}
$$

where $C_{t}=\int_{0}^{t} c(s) \mathrm{d} s$. Since $\left\|\mathbf{e}_{h}(t)\right\|=\sum_{i=1}^{2 N+1}\left|e\left(t, x_{i}\right)\right| h$, the above indicates that

$$
e\left(t, x_{i}\right)=O\left(h^{2}\right), \quad \text { for all } i=1, \ldots, 2 N+1, t \in[0, T],
$$

which was our claim.

Remark 3. The above proposition assumes that $X_{t}$ and $X_{t}^{h}$ have the same initial probability measure. In the special case $X_{0}^{h}=X_{0}=x_{0} \in \mathcal{X}$, the fact that the PDF of $X_{0}$ is a nondifferentiable delta function will cause some technical problems, i.e. the initial error $\mathbf{e}_{h}(0)$ will become unbounded even though they start from the same value $x_{0}$. But the problem is eased after a small $\Delta t$ at which time the PDF of $X_{\Delta t}$ is normally distributed around $x_{0}$ with a small variance. The above proposition is applicable by taking $\Delta t$ to be the initial time.

Remark 4. The second order convergence is clearly due to the fact that the first two moments are matched. If more moments are matched (i.e. transitions to other states are allowed, not just to the adjacent states), the convergence will generally become quicker, but this will not necessarily improve the order of convergence. 


\section{Closed-form formula of the moment generating function}

In this section we derive the moment generating function of the discrete OU process. We first discuss how to formulate the PDE which the MGF of $X_{t}^{h}$ should satisfy. Then we present its closed-form solution. Finally we use this result to examine the convergence order.

\subsection{Formulation and solution of the PDE for the MGF of $\boldsymbol{X}_{\boldsymbol{t}}^{\boldsymbol{h}}$}

Since the MGF takes the form of an expected value, it is of interest to look at a result about general expected values. The following proposition provides the differential equation for $\mathbb{E}\left[f\left(X_{t}^{h}\right)\right]$.

Proposition 4. For a function $f \in \mathbf{C}^{\infty}(\mathbb{R}), \mathbb{E}\left[f\left(X_{t}^{h}\right)\right]$ must solve the following differential equation

$$
\begin{aligned}
\frac{\mathrm{d} \mathbb{E}\left[f\left(X_{t}^{h}\right)\right]}{\mathrm{d} t}= & \mathbb{E}\left[k\left(\theta-X_{t}^{h}\right) f^{\prime}\left(X_{t}^{h}\right)\right]+\frac{1}{2} \mathbb{E}\left[\sigma^{2} f^{\prime \prime}\left(X_{t}^{h}\right)\right] \\
& +\frac{h^{2}}{3 !} \mathbb{E}\left[k\left(\theta-X_{t}^{h}\right) f^{(3)}\left(X_{t}^{h}\right)\right]+\frac{h^{2}}{4 !} \mathbb{E}\left[\sigma^{2} f^{(4)}\left(X_{t}^{h}\right)\right] \\
& +\frac{h^{4}}{5 !} \mathbb{E}\left[k\left(\theta-X_{t}^{h}\right) f^{(5)}\left(X_{t}^{h}\right)\right]+\frac{h^{4}}{6 !} \mathbb{E}\left[\sigma^{2} f^{(6)}\left(X_{t}^{h}\right)\right]+\cdots .
\end{aligned}
$$

Proof. Define the generator for the discrete OU process $X_{t}^{h}$, denoted $\mathcal{A}_{h}$, as

$$
\mathcal{A}_{h} f(x)=\lim _{\Delta t \rightarrow 0} \frac{\mathbb{E}\left[f\left(X_{t+\Delta t}^{h}\right) \mid X_{t}^{h}=x\right]-f(x)}{\Delta t}
$$

for a function $f \in \mathbf{C}^{\infty}(\mathbb{R})$ and for all $x \in \mathcal{X}$. Respectively let $u$ and $d$ be the up and down jump rates for the state $x$ (subscript $i$ dropped), then

$\mathbb{E}\left[f\left(X_{t+\Delta t}^{h}\right) \mid X_{t}^{h}=x\right]=(u \Delta t) f(x+h)+(1-(u+d) \Delta t) f(x)+(d \Delta t) f(x-h)+O\left((\Delta t)^{2}\right)$.

Consequently, the generator in (12) becomes

$$
\begin{aligned}
\mathcal{A}_{h} f(x) & =u f(x+h)-(u+d) f(x)+d f(x-h) \\
& =\left[\frac{\sigma^{2}+h k(\theta-x)}{2 h^{2}}\right] f(x+h)-\left[\frac{\sigma^{2}}{h^{2}}\right] f(x)+\left[\frac{\sigma^{2}-h k(\theta-x)}{2 h^{2}}\right] f(x-h) \\
& =k(\theta-x)\left[\frac{f(x+h)-f(x-h)}{2 h}\right]+\frac{1}{2} \sigma^{2}\left[\frac{f(x+h)-2 f(x)+f(x-h)}{h^{2}}\right] .
\end{aligned}
$$

Applying Taylor expansions to $f(x+h)$ and $f(x-h)$ in (13) yields

$$
\begin{aligned}
\mathcal{A}_{h} f(x)= & k(\theta-x)\left[f^{\prime}(x)+\frac{f^{(3)}(x)}{3 !} h^{2}+\frac{f^{(5)}(x)}{5 !} h^{4}+\cdots\right] \\
& +\sigma^{2}\left[\frac{f^{\prime \prime}(x)}{2 !}+\frac{f^{(4)}(x)}{4 !} h^{2}+\frac{f^{(6)}(x)}{6 !} h^{4}+\cdots\right] .
\end{aligned}
$$

Note that (12) can be expressed as

$$
\mathbb{E}\left[f\left(X_{t+\Delta t}^{h}\right) \mid X_{t}^{h}=x\right]=f(x)+\mathscr{A}_{h} f(x) \Delta t+o(\Delta t) .
$$


Taking the expected value with respect to $X_{t}^{h}$, we have

$$
\mathbb{E}\left[f\left(X_{t+\Delta t}^{h}\right)\right]=\mathbb{E}\left[f\left(X_{t}^{h}\right)\right]+\mathbb{E}\left[\mathcal{A}_{h} f\left(X_{t}^{h}\right)\right] \Delta t+o(\Delta t) .
$$

Inserting $\mathcal{A}_{h}$ into the above formula and letting $\Delta t \rightarrow 0$, we obtain (11).

In order to find the MGF, we set $f(x)=\mathrm{e}^{\alpha x}$ in (11) and obtain

$$
\begin{aligned}
\frac{\mathrm{d} \mathbb{E}\left[\mathrm{e}^{\alpha X_{t}^{h}}\right]}{\mathrm{d} t}= & \mathbb{E}\left[k\left(\theta-X_{t}^{h}\right) \alpha \mathrm{e}^{\alpha X_{t}^{h}}\right]+\frac{1}{2} \mathbb{E}\left[\sigma^{2} \alpha^{2} \mathrm{e}^{\alpha X_{t}^{h}}\right] \\
& +\frac{h^{2}}{3 !} \mathbb{E}\left[k\left(\theta-X_{t}^{h}\right) \alpha^{3} \mathrm{e}^{\alpha X_{t}^{h}}\right]+\frac{h^{2}}{4 !} \mathbb{E}\left[\sigma^{2} \alpha^{4} \mathrm{e}^{\alpha X_{t}^{h}}\right]+\cdots
\end{aligned}
$$

From an initial look of (14), the presence of the infinite terms on the right-hand side seems to pose some problem. However, some algebraic manipulations turn the differential equation (14) into a simple first order PDE, of which the closed-form solution can be found. The following proposition presents the results.

Proposition 5. The MGF of $X_{t}^{h}$, denoted as $y=y(t, \alpha)=\mathbb{E}\left[\mathrm{e}^{\alpha X_{t}^{h}}\right]$, satisfies the following PDE:

$$
\frac{\partial y}{\partial t}+\left[\frac{k}{h} \sinh (\alpha h)\right] \frac{\partial y}{\partial \alpha}=\left[\frac{k \theta}{h} \sinh (\alpha h)+\frac{\sigma^{2}}{h^{2}}(\cosh (\alpha h)-1)\right] y .
$$

Its solution is given by

$$
\mathbb{E}\left[\mathrm{e}^{\alpha X_{t}^{h}}\right]=\mathrm{e}^{\alpha \theta+(2 / h)\left(x_{0}-\theta\right) \tanh ^{-1}\left(\mathrm{e}^{-k t} \tanh (\alpha h / 2)\right)}\left[\frac{\cosh (\alpha h / 2)}{\cosh \left(\tanh ^{-1}\left(\mathrm{e}^{-k t} \tanh (\alpha h / 2)\right)\right)}\right]^{2 \sigma^{2} / h^{2} k} .
$$

Proof. Since $\partial \mathbb{E}\left[\mathrm{e}^{\alpha X_{t}^{h}}\right] / \partial \alpha=\mathbb{E}\left[X_{t}^{h} \mathrm{e}^{\alpha X_{t}^{h}}\right],(14)$ can be written as a first order PDE where the derivatives are with respect to either $t$ or $\alpha$. By collecting the $k$ and $\sigma^{2}$ related terms on the right-hand side of (14) and using the following two formulas

$$
1+\frac{1}{3 !}(\alpha h)^{2}+\frac{1}{5 !}(\alpha h)^{4}+\cdots=\frac{\sinh (\alpha h)}{\alpha h}, \quad 1+\frac{2}{4 !}(\alpha h)^{2}+\frac{2}{6 !}(\alpha h)^{4}+\cdots=2 \frac{\cosh (\alpha h)-1}{(\alpha h)^{2}},
$$

the first order PDE can be simplified to (15). The details of solving (15) to derive its solution (16) are provided in Appendix A.

\subsection{Convergence from the MGF of $X_{t}^{h}$ to the MGF of $X_{t}$}

We may use (16) to study the convergence $\mathbb{E}\left[\mathrm{e}^{\alpha X_{t}^{h}}\right] \rightarrow \mathbb{E}\left[\mathrm{e}^{\alpha X_{t}}\right]$. Recall that $X_{t}$ is normally distributed with mean $\theta+\left(x_{0}-\theta\right) \mathrm{e}^{-k t}$, variance $\left(\sigma^{2} / 2 k\right)\left(1-\mathrm{e}^{-2 k t}\right)$ (see [12]), and MGF

$$
\mathbb{E}\left[\mathrm{e}^{\alpha X_{t}}\right]=\mathrm{e}^{\alpha\left[\theta+\left(x_{0}-\theta\right) \mathrm{e}^{-k t}\right]+\left(\alpha^{2} / 2\right)\left[\left(\sigma^{2} / 2 k\right)\left(1-\mathrm{e}^{-2 k t}\right)\right]} .
$$

The order of convergence is checked in the following proposition.

Proposition 6. For an $\alpha$ such that both MGFs exist, $\mathbb{E}\left[\mathrm{e}^{\alpha X_{t}^{h}}\right]$ converges to $\mathbb{E}\left[\mathrm{e}^{\alpha X_{t}}\right]$ in second order, i.e.

$$
\mathbb{E}\left[\mathrm{e}^{\alpha X_{t}^{h}}\right]=\mathbb{E}\left[\mathrm{e}^{\alpha X_{t}}\right]\left(1+O\left(h^{2}\right)\right) .
$$


Proof. We check the convergence order of the following terms in (16) separately:

$$
\begin{gathered}
(\mathrm{A})=\mathrm{e}^{(2 / h)\left(x_{0}-\theta\right) \tanh ^{-1}\left(\mathrm{e}^{-k t} \tanh (\alpha h / 2)\right)} \longrightarrow(\mathrm{B})=\mathrm{e}^{\alpha\left(x_{0}-\theta\right) \mathrm{e}^{-k t}}, \\
(\mathrm{C})=\left[\frac{\cosh (\alpha h / 2)}{\cosh \left(\tanh ^{-1}\left(\mathrm{e}^{-k t} \tanh (\alpha h / 2)\right)\right)}\right]^{2 \sigma^{2} / h^{2} k} \longrightarrow(\mathrm{D})=\mathrm{e}^{\left(\alpha^{2} / 2\right)\left[\left(\sigma^{2} / 2 k\right)\left(1-\mathrm{e}^{-2 k t}\right)\right]} .
\end{gathered}
$$

Using the relation $\tanh (x) \approx x \approx \tanh ^{-1}(x)$ for sufficiently small $x$, we see that as $h \rightarrow 0$,

$$
\frac{2\left(x_{0}-\theta\right)}{h} \tanh ^{-1}\left(\mathrm{e}^{-k t} \tanh \left(\frac{\alpha h}{2}\right)\right) \longrightarrow \frac{2\left(x_{0}-\theta\right)}{h} \mathrm{e}^{-k t}\left(\frac{\alpha h}{2}\right)=\alpha\left(x_{0}-\theta\right) \mathrm{e}^{-k t},
$$

which shows that (A) converges to (B). To check the convergence order, note that

$$
\tanh (x)=\frac{\mathrm{e}^{x}-\mathrm{e}^{-x}}{\mathrm{e}^{x}+\mathrm{e}^{-x}}=\frac{\left(1+x+x^{2} / 2+\cdots\right)-\left(1-x+x^{2} / 2-\cdots\right)}{\left(1+x+x^{2} / 2+\cdots\right)+\left(1-x+x^{2} / 2-\cdots\right)}=x+O\left(x^{3}\right),
$$

and

$$
\begin{aligned}
\tanh ^{-1}(x) & =\frac{1}{2} \ln \left(\frac{1+x}{1-x}\right) \\
& =\frac{1}{2} \ln \left[(1+x)\left(1+x+x^{2}+\cdots\right)\right] \\
& =\frac{1}{2} \ln \left(1+2 x+2 x^{2}+2 x^{3}+\cdots\right) \\
& =\frac{1}{2}\left[\left(2 x+2 x^{2}+2 x^{3}+\cdots\right)-\frac{\left(2 x+2 x^{2}+2 x^{3}+\cdots\right)^{2}}{2}+\cdots\right] \\
& =x+O\left(x^{3}\right) .
\end{aligned}
$$

As a result,

$$
\begin{aligned}
\frac{2\left(x_{0}-\theta\right)}{h} \tanh ^{-1}\left(\mathrm{e}^{-k t} \tanh \left(\frac{\alpha h}{2}\right)\right) & =\frac{2\left(x_{0}-\theta\right)}{h}\left[\mathrm{e}^{-k t}\left(\frac{\alpha h}{2}\right)+O\left(h^{3}\right)\right] \\
& =\alpha\left(x_{0}-\theta\right) \mathrm{e}^{-k t}+O\left(h^{2}\right)
\end{aligned}
$$

Thus we may write $(\mathrm{A})=(\mathrm{B})\left(1+O\left(h^{2}\right)\right)$. Next, to see how $(\mathrm{C})$ converges to $(\mathrm{D})$, we use

$$
\begin{aligned}
{[\cosh (a x)]^{y / x^{2}}=} & {\left[\left(\frac{\mathrm{e}^{a x}+\mathrm{e}^{-a x}}{2}\right)^{1 / x^{2}}\right]^{y} } \\
= & {\left[\left(1+\frac{a^{2}}{2} x^{2}+\cdots\right)^{1 / x^{2}}\right]^{y} } \\
& \longrightarrow\left[\mathrm{e}^{a^{2} / 2}\right]^{y} \\
= & \mathrm{e}^{a^{2} y / 2} .
\end{aligned}
$$

Also note that

$$
\mathrm{e}^{a^{2} / 2}-\left(1+\frac{a^{2}}{2} x^{2}+\cdots\right)^{1 / x^{2}}=O\left(x^{2}\right)
$$

thus

$$
[\cosh (a x)]^{y / x^{2}}=\mathrm{e}^{a^{2} y / 2}\left(1+O\left(x^{2}\right)\right) .
$$


As a result,

$$
\begin{gathered}
{\left[\cosh \left(\frac{\alpha h}{2}\right)\right]^{2 \sigma^{2} / h^{2} k}=\mathrm{e}^{\left(\alpha^{2} / 2\right)\left(\sigma^{2} / 2 k\right)}\left(1+O\left(h^{2}\right)\right),} \\
{\left[\cosh \left(\tanh ^{-1}\left(\mathrm{e}^{-k t} \tanh \left(\frac{\alpha h}{2}\right)\right)\right)\right]^{2 \sigma^{2} / h^{2} k}=\mathrm{e}^{\left(\alpha^{2} / 2\right)\left(\sigma^{2} / 2 k\right) \mathrm{e}^{-2 k t}}\left(1+O\left(h^{2}\right)\right) .}
\end{gathered}
$$

Consequently, we have $(\mathrm{C})=(\mathrm{D})\left(1+O\left(h^{2}\right)\right)$. Putting all these together, we see the overall second order convergence.

\section{Steady state analysis}

In the preceding section we derived the explicit form for the MGF of $X_{t}^{h}$ for any time horizon $t$. One may wonder whether it is possible to derive the explicit form of the probability distribution (probability mass function) of $X_{t}^{h}$. In fact, for $t<\infty$ (transient state), it is not easy to obtain the distribution function analytically. On the other hand, when $t \rightarrow \infty$ (steady state), the distribution of $X_{t}^{h}$ is particularly tractable. In this section, we will give a full characterisation of the steady state probability distribution.

Let us define $X=\lim _{t \rightarrow \infty} X_{t}$ and $X_{h}=\lim _{t \rightarrow \infty} X_{t}^{h}$ to lighten notations. (Clearly $X$ and $X_{h}$ do not depend on their initial measures.) The steady state analysis relies on the existence of equilibrium, i.e. the limiting probability distribution is stationary. For the continuous OU process, it is known that the equilibrium state exists and $X$ follows a normal distribution. It can be reasonably expected that such an equilibrium also exists for the discrete OU process. The following proposition gives the main result derived from the existence of equilibrium (which is assumed here and can be easily justified later). It shows that $X_{h}$ actually follows the same distribution as an affine function of a binomial random variable.

Proposition 7. For the discrete OU process $X_{t}^{h}$, assuming the existence of equilibrium when $t \rightarrow \infty, X_{h}$ follows a scaled and shifted binomial distribution and has the same distribution as the random variable

$$
Y=h Z+(\theta-N h),
$$

where $Z$ is a binomial random variable with parameters $\operatorname{Bin}\left(2 N, \frac{1}{2}\right)$.

Proof. Firstly, we show that $X_{h}$ and $Y$ take values from the same set. The domain set of $X_{h}$ is $\mathcal{X}=\left\{x_{i}\right\}$ where

$$
x_{i}=x_{1}+(i-1) h=h(i-1)+(\theta-N h), \quad i=1, \ldots, 2 N+1 .
$$

On the other hand, since $Y=h Z+(\theta-N h)$, where $Z=0, \ldots, 2 N$, it is obvious that $Y$ also takes values from $\mathcal{X}$. To prove the claimed result, we need to show $\mathbb{P}\left(X_{h}=x_{i}\right)=\mathbb{P}\left(Y=x_{i}\right)$ for all $i$, where

$$
\mathbb{P}\left(Y=x_{i}\right)=\mathbb{P}(Z=i-1)=\left(\begin{array}{c}
2 N \\
i-1
\end{array}\right)\left(\frac{1}{2}\right)^{2 N}, \quad i=1, \ldots, 2 N+1 .
$$

Let $\mathbb{P}\left(X_{h}=x_{i}\right)=p_{i}$ for simplicity. By equilibrium, when $t \rightarrow \infty$, the adjacent state probabilities should follow the local balance equations as $p_{i} u_{i}=p_{i+1} d_{i+1}, i=1, \ldots, 2 N$. Using (2), (4), and (5), we have $u_{i}, d_{i}=\left(\sigma^{2} / h^{2}\right)(1 \pm(N+1-i) / N)$ and these balance equations become

$$
p_{i}(2 N+1-i)=p_{i+1}(i), \quad i=1, \ldots, 2 N .
$$


Because of the symmetry between the first half states $(i<N+1)$ and the second half states $(i>N+1)$, we have $p_{N+1-j}=p_{N+1+j}, j=1, \ldots, N$. Thus we only need to derive either half of these state probabilities. For states $i \geq N+1$, the balance equations can be written as

$$
p_{N+j}(N-j+1)=p_{N+1+j}(N+j), \quad j=1, \ldots, N .
$$

Let $p_{N+1}=p$. We may recursively calculate all the state probabilities using

$$
p_{N+1-j}=p_{N+1+j}=p \frac{N}{N+1} \frac{N-1}{N+2} \cdots \frac{N-j+1}{N+j}, \quad j=1, \ldots, N .
$$

By setting the sum of these probabilities to $1, p$ is found to be $p=\left(\begin{array}{c}2 N \\ N\end{array}\right)\left(\frac{1}{2}\right)^{2 N}$, and therefore

$$
\mathbb{P}\left(X_{h}=x_{i}\right)=p_{i}=\left(\begin{array}{c}
2 N \\
i-1
\end{array}\right)\left(\frac{1}{2}\right)^{2 N}, \quad i=1, \ldots, 2 N+1,
$$

which completes the proof.

Since we have fully characterised the distribution of $X_{h}$, its MGF and moments can be obtained without difficulty. The next proposition presents the results.

Proposition 8. (i) The $M G F$ of $X_{h}$ is given by

$$
\mathbb{E}\left[\mathrm{e}^{\alpha X_{h}}\right]=\mathrm{e}^{\alpha \theta}\left[\frac{\mathrm{e}^{\alpha h / 2}+\mathrm{e}^{-\alpha h / 2}}{2}\right]^{2 N} \quad\left(=\mathrm{e}^{\alpha \theta}\left[\cosh \left(\frac{\alpha h}{2}\right)\right]^{2 N}\right) .
$$

(ii) The first four moments of $X_{h}$ are given by

$$
\begin{gathered}
\mathbb{E}\left[X_{h}\right]=\theta, \quad \mathbb{E}\left[X_{h}^{2}\right]=\theta^{2}+\frac{\sigma^{2}}{2 k}, \\
\mathbb{E}\left[X_{h}^{3}\right]=\theta^{3}+3 \theta\left(\frac{\sigma^{2}}{2 k}\right), \quad \mathbb{E}\left[X_{h}^{4}\right]=\theta^{4}+6 \theta^{2}\left(\frac{\sigma^{2}}{2 k}\right)+3\left(\frac{\sigma^{2}}{2 k}\right)^{2}-h^{2}\left(\frac{\sigma^{2}}{4 k}\right) .
\end{gathered}
$$

Proof. Since $Y=h Z+(\theta-N h)$, where $Z \sim \operatorname{Bin}\left(2 N, \frac{1}{2}\right)$ (whose MGF is given by $\left.\mathbb{E}\left[\mathrm{e}^{\alpha Z}\right]=\left[\left(1+\mathrm{e}^{\alpha}\right) / 2\right]^{2 N}\right)$, the claimed MGF is obtained as

$$
\begin{aligned}
\mathbb{E}\left[\mathrm{e}^{\alpha X_{h}}\right] & =\mathbb{E}\left[\mathrm{e}^{\alpha Y}\right] \\
& =\mathbb{E}\left[\mathrm{e}^{\alpha h Z}\right] \mathrm{e}^{\alpha(\theta-N h)} \\
& =\left[\frac{1+\mathrm{e}^{\alpha h}}{2}\right]^{2 N} \mathrm{e}^{\alpha(\theta-N h)} \\
& =\mathrm{e}^{\alpha \theta}\left[\frac{\mathrm{e}^{\alpha h / 2}+\mathrm{e}^{-\alpha h / 2}}{2}\right]^{2 N} .
\end{aligned}
$$

To find the moments of $X_{h}$, we first use $\mathbb{E}\left[Z^{n}\right]=\left.\left(\mathrm{d}^{n} / \mathrm{d} \alpha^{n}\right) \mathbb{E}\left[\mathrm{e}^{\alpha Z}\right]\right|_{\alpha=0}$ to find the moments of $Z$ as

$$
\begin{gathered}
\mathbb{E}[Z]=N, \quad \mathbb{E}\left[Z^{2}\right]=N^{2}+\frac{1}{2} N, \\
\mathbb{E}\left[Z^{3}\right]=N^{3}+\frac{1}{2} N^{2}+\frac{1}{2} N, \quad \mathbb{E}\left[Z^{4}\right]=N^{4}+3 N^{3}+\frac{3}{4} N^{2}-\frac{1}{4} N .
\end{gathered}
$$


Then the first four moments of $X_{h}$ can be found from its relationship with $Y$ and $Z$, i.e.

$$
\mathbb{E}\left[X_{h}^{n}\right]=\mathbb{E}\left[Y^{n}\right]=\mathbb{E}\left[(h Z+\theta-N h)^{n}\right], \quad n=1,2,3,4 .
$$

After some further derivations, the claimed results are obtained.

We may use the MGF given in (18) to examine the existence of the equilibrium which is assumed previously. It is observed that as $t \rightarrow \infty$, the result in (16) will coincide with (18). This agreement indicates that the equilibrium based analysis leads to the correct result. Another way of checking its correctness is to examine whether the limiting characteristic function $\phi(\alpha)=$ $\lim _{t \rightarrow \infty} \phi(\alpha, t)$ is continuous at $\alpha=0$ where $\phi(\alpha, t)=\mathbb{E}\left[\mathrm{e}^{i \alpha X_{t}^{h}}\right]$ (see, e.g. the continuity theorem in [3, p. 172]). This can be easily seen from (18).

Once again we may use the results in Proposition 8 to check the convergence order. This is clear for the moments (see the $h^{2}\left(\sigma^{2} / 4 k\right)$ term in $\mathbb{E}\left[X_{h}^{4}\right]$; the fact that there is no $h$-related term in $\mathbb{E}\left[X_{h}^{n}\right], n \leq 3$, means that these moments already match $\mathbb{E}\left[X^{n}\right]$ exactly). For the MGF, the convergence is observed below

$$
\begin{aligned}
\mathbb{E}\left[\mathrm{e}^{\alpha X_{h}}\right]= & \mathrm{e}^{\alpha \theta}\left[1+\frac{1}{2 !}\left(\frac{\alpha h}{2}\right)^{2}+\frac{1}{4 !}\left(\frac{\alpha h}{2}\right)^{4}+\cdots\right]^{2 \sigma^{2} / h^{2} k} \\
= & \mathrm{e}^{\alpha \theta}\left[\left(1+\frac{\alpha^{2}}{8} h^{2}+o\left(h^{2}\right)\right)^{1 / h^{2}}\right]^{2 \sigma^{2} / k} \\
& \stackrel{h \rightarrow 0}{\longrightarrow} \mathrm{e}^{\alpha \theta+\left(\alpha^{2} / 2\right)\left(\sigma^{2} / 2 k\right)} \\
= & \mathbb{E}\left[\mathrm{e}^{\alpha X}\right]
\end{aligned}
$$

(see (17) for the last equality.) We note that $h^{2}$ is involved in the expression of $\mathbb{E}\left[\mathrm{e}^{\alpha X_{h}}\right]$, confirming the second order convergence.

\section{Conclusions}

Motivated by the discrete nature found in the stochastic processes for financial markets, we consider the discrete OU process and conduct its analysis. This discrete process is constructed by matching the first two moments across an infinitesimal interval. Our analysis leads to three main results about the discrete OU process. Firstly, the convergence speed from the discrete to the continuous processes is in the second order of step size. Secondly, the moment generating function for any time horizon and step size is derived in closed-form. Thirdly, the steady state probability distribution is completely characterised. The order of convergence can also be observed from the latter two analytical results.

\section{Appendix A. Solving (15) to derive the MGF}

Equation (15) is a special kind of first order PDE known as a quasilinear PDE (see [4] and [5]), for which the general form is $p(\partial y / \partial t)+q(\partial y / \partial x)=r$ and the characteristic equation is

$$
\frac{\mathrm{d} t}{p}=\frac{\mathrm{d} x}{q}=\frac{\mathrm{d} y}{r} .
$$

The approach to solving this PDE is described as follows. Solving the first two terms (first equality) in the characteristic equation gives a constant of integration $c_{1}$, while solving the latter two (second equality) gives another constant of integration $c_{2}$. The main technique is to set 
$g\left(c_{1}\right)=c_{2}$ and find the function $g(\cdot)$. Then the solution of $y$ can be obtained from the constants $c_{1}, c_{2}$ and the function $g(\cdot)$.

Applying this idea to (15), we express the characteristic equation as

$$
\frac{\mathrm{d} t}{1}=\frac{\mathrm{d} \alpha}{(k / h) \sinh (\alpha h)}=\frac{\mathrm{d} y}{\left[(k \theta / h) \sinh (\alpha h)+\left(\sigma^{2} / h^{2}\right)(\cosh (\alpha h)-1)\right] y} .
$$

By applying the formula

$$
\int \frac{1}{\sinh (x)} \mathrm{d} x=\ln \tanh \left(\frac{x}{2}\right)+c
$$

to the equation formed by the first equality in (19), we have

$$
t=\frac{h}{k} \int \frac{1}{\sinh (\alpha h)} \mathrm{d} \alpha=\frac{1}{k} \ln \tanh \left(\frac{\alpha h}{2}\right)+c_{1} \quad \Longrightarrow \quad c_{1}=\mathrm{e}^{-k t} \tanh \left(\frac{\alpha h}{2}\right) .
$$

Now we look at the equation formed by the second equality in (19)

$$
\frac{\mathrm{d} \ln y}{\mathrm{~d} \alpha}=\frac{(k \theta / h) \sinh (\alpha h)+\left(\sigma^{2} / h^{2}\right)(\cosh (\alpha h)-1)}{(k / h) \sinh (\alpha h)}=\theta+\frac{\sigma^{2}}{h k} \tanh \left(\frac{\alpha h}{2}\right) .
$$

We use another formula

$$
\int \tanh (x) \mathrm{d} x=\ln \cosh (x)+c
$$

to obtain

$$
\ln y=\alpha \theta+\frac{2 \sigma^{2}}{h^{2} k} \ln \cosh \left(\frac{\alpha h}{2}\right)+c_{2} \quad \Longrightarrow \quad c_{2}=\frac{y}{\mathrm{e}^{\alpha \theta}[\cosh (\alpha h / 2)]^{2 \sigma^{2} / h^{2} k}} .
$$

Now we apply the main technique to set $g\left(c_{1}\right)=c_{2}$, i.e.

$$
g\left(\mathrm{e}^{-k t} \tanh \left(\frac{\alpha h}{2}\right)\right)=\frac{y}{\mathrm{e}^{\alpha \theta}[\cosh (\alpha h / 2)]^{2 \sigma^{2} / h^{2} k}} .
$$

Remember that our target is the function $y=y(t, \alpha)$. Suppose that the initial condition is given as $y(0, \alpha)=y_{0}(\alpha)=\mathbb{E}\left[\mathrm{e}^{\alpha X_{0}^{h}}\right]$ (i.e. the initial measure of $X_{0}^{h}$ and its MGF are assumed to be given). Setting $t=0$ in the above equation gives

$$
g\left(\tanh \left(\frac{\alpha h}{2}\right)\right)=\frac{y_{0}(\alpha)}{\mathrm{e}^{\alpha \theta}[\cosh (\alpha h / 2)]^{2 \sigma^{2} / h^{2} k}} .
$$

Define $v=\tanh (\alpha h / 2)$ and, thus, $\alpha=(2 / h) \tanh ^{-1}(v)$, then the function $g(\cdot)$ can be found as

$$
g(v)=\frac{y_{0}\left((2 / h) \tanh ^{-1}(v)\right)}{\mathrm{e}^{(2 / h) \tanh ^{-1}(v) \theta}\left[\cosh \left(\tanh ^{-1}(v)\right)\right]^{2 \sigma^{2} / h^{2} k}} .
$$

Using $c_{2}=g\left(c_{1}\right)$ with $c_{1}=\mathrm{e}^{-k t} \tanh (\alpha h / 2)$, we obtain $c_{2}$ as a function of $t$ and $\alpha$. By further substituting this $c_{2}$ into (20), we arrive at the final closed-form formula for $y$ as

$$
\begin{aligned}
& y(t, \alpha)=\mathbb{E}\left[\mathrm{e}^{\alpha X_{t}^{h}}\right]
\end{aligned}
$$

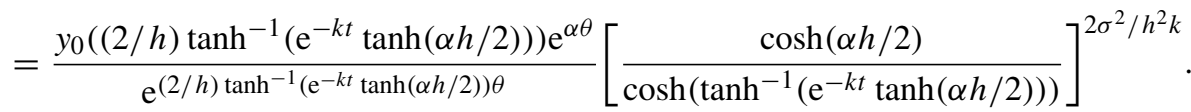

The equation (16) is a special case when $X_{0}^{h}=x_{0}$ (i.e. $X_{t}^{h}$ has a Dirac measure as its initial measure) and $y_{0}(\alpha)=\mathrm{e}^{\alpha x_{0}}$. 


\section{Acknowledgements}

The author is very grateful to the anonymous referee for providing valuable comments on the improvements to this paper. In addition, the author would like to thank Professor Ben M. Hambly (Mathematical Institute, University of Oxford, UK) for his supervision and guidance on this research project, and Professor Chii-Ruey Hwang (Institute of Mathematics, Academia Sinica, Taiwan) for the helpful discussions.

\section{References}

[1] Black, F. and Scholes, M. (1973). The pricing of options and corporate liabilites. J. Political Econom. 81, 637-654.

[2] Chung, S.-L. and Shin, P.-T. (2007). Generalized Cox-Ross-Rubinstein binomial models. Manag. Sci. 53, 508-520.

[3] Grimmett, G. R. and Stirzaker, D. R. (1992). Probability and Random Processes, 2nd edn. Oxford University Press.

[4] Gross, D. And Harris, C. M. (1985). Fundamentals of Queueing Theory, 2nd edn. John Wiley, New York.

[5] Howison, S. (2005). Practical Applied Mathematics, Modeling, Analysis, Approximation. Cambridge University Press.

[6] Hull, J. And White, A. (1990). Pricing interest rate derivative securities. Rev. Financial Studies 3, $573-592$.

[7] Larralde, H. (2004). A first passage time distribution for a discrete version of the Ornstein-Uhlenbeck process. J. Phys. A 37, 3759-3767.

[8] Larralde, H. (2004). Statistical properties of a discrete version of the Ornstein-Uhlenbeck process. Physical Rev. E 69, 027102.

[9] Lefebvre, M. and Guilbault, J.-L. (2009). First hitting place probabilities for a discrete version of the Ornstein-Uhlenbeck process. Internat. J. Math. Math. Sci. 2009, 909835.

[10] Renshaw, E. (1987). The discrete Uhlenbeck-Ornstein process. J. Appl. Prob. 24, 908-917.

[11] Uhlenbeck, G. E. And Ornstein, L. S. (1930). On the theory of Brownian motion. Physical Rev. 36, 823-841.

[12] VASIČEK, O. (1977). An equilibrium characterization of the term structure. J. Financial Econom. 5, 177-188.

[13] WaLsh, J. B. (2003). The rate of convergence of the binomial tree scheme. Finance Stoch. 7, 337-361. 